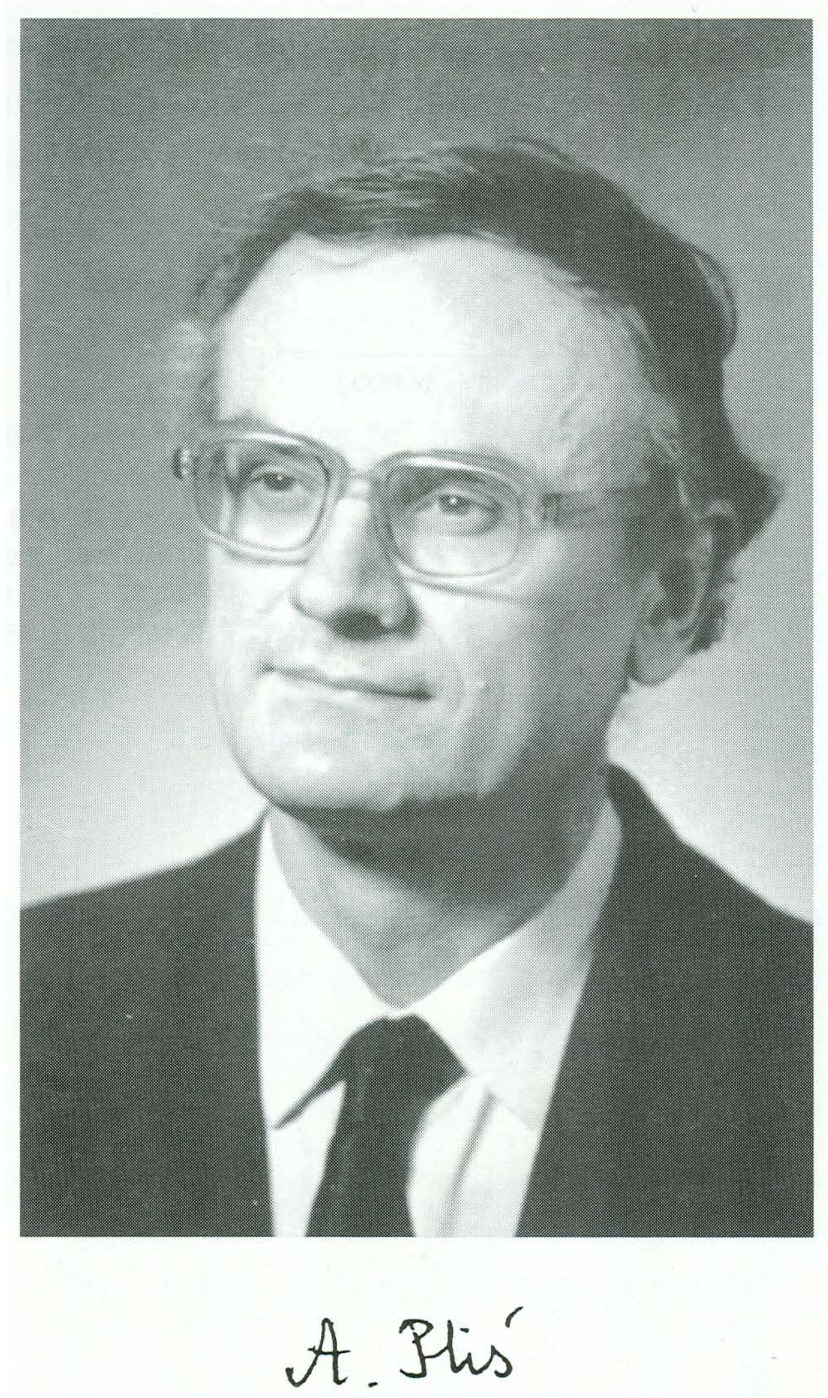




\title{
ANDRZEJ PLIŚ
}

\author{
(17.3.1929-28.11.1991)
}

Andrzej Pliś pursued his academic and teaching activities at the Jagiellonian University and at the Mathematical Institute of the Polish Academy of Sciences.

He studied at the Jagiellonian University in Cracow in the years 1947-51. His first steps in mathematical research were under Tadeusz Ważewski. He received his Ph.D. in 1954.

In 1961 he was promoted to associate professor and in 1966 to full professor. Since 1976 he was a member of the Polish Academy of Sciences.

Andrzej Pliś published about 60 papers during his lifetime. He investigated various problems in the theory of differential equations, one of the most important and oldest mathematical domains. The results of Pliś are among the most eminent in the field. He solved the extremely difficult problem of uniqueness of solutions of the Cauchy problem for general linear elliptic partial differential operators with non-analytic coefficients, stated as far back as 1901 by Holmgren. His results on this subject were expounded at the prestigious seminars of Bourbaki and Schwartz in Paris, at which only the most important achievements used to be presented. Now these results are regarded as classical and can be found in the most important books on the subject, in particular in Hörmander's monograph. Lars Hörmander, a Fields medalist, recently created new theories based on an analysis of Pliś's results. Another Fields medalist who took up Pliś's results is Paul Cohen. Because of the importance of his work Pliś was invited to present a plenary lecture at the International Congress of Mathematicians in Stockholm in 1962 .

In the theory of characteristics of non-linear partial differential equations of the first order, Pliś solved another old and difficult problem on generating continuously differentiable solutions by characteristics. This problem was attacked as far back as 1929 by Haar. Pliś's proof is surprisingly fine.

Pliś was one of the deepest analysts in the world. 
The concept of the second order characteristic band that he invented led to important theorems on the existence and evaluation of domains of solutions of first order partial differential equations.

In optimal control theory Pliś's results are also well known. The new methods he invented became a starting point for the research of other mathematicians. They include: a characterization of the measurability of an orientor field, results on Carathéodory conditions and on the Pontryagin principle, and a particularly useful theorem stating that the Lipschitz condition implies the bang-bang type.

Another domain of his activity was the theory of approximation of solutions of differential equations by means of difference algorithms. His fundamental results on higher order precision convex schemes for non-linear partial differential equations initiated new lines of research, carried on by specialists of international importance.

Pliś was deeply interested in mathematical physics. He discovered some fundamental relations between the Feynman integral and the Wiener measure.

Pliś was highly appreciated as a teacher by his students as well as by his colleagues for his expert advice. Because of his influence on other mathematicians and his particular gift for communicating with and caring for others, he became a person of great importance in our mathematical community. Seven of his pupils wrote their Ph.D. theses under his supervision and four of them have already done their habilitations. On his initiative Bolesław Szafirski created an active center of probabilistic turbulence theory in Cracow.

Also in Mexico, where Pliś spent a period of two years, his merits in the development of mathematics in that country are highly appreciated. One of his students, Roberto Suarez, was elected to the Mexican Academy of Sciences.

Andrzej Pliś was a member of the editorial board of our Journal and of some other scientific periodicals, including the important Journal of Differential Equations.

He was the head of the Cracow Branch of the Mathematical Institute of the Polish Academy of Sciences, and of the Chair of Probability Theory at the Jagiellonian University in Cracow. He was the President of the Cracow Branch of the Polish Mathematical Society. For many years he presided over the Committee for Fundamental Research Problems in Poland and also the Committee for Education in Applied Mathematics in Poland.

The death of Andrzej Pliś is a painful loss to the Polish mathematical community, and no-one will be able to take his place. As a mathemati- 
cian, the late Andrzej Pliś was one of the most outstanding analysts on the world-wide scale. But he was also a magnificent personality, with a profound understanding both of professional and organizational matters, as well as of personal, human concerns. He was a wonderful friend, teacher and father. We miss him.

\section{S. Eojasiewicz}

